\title{
3D CFD Modeling of Air Flow Through a Porous Fence
}

\author{
${ }^{1}$ Yizhong Xu, ${ }^{1}$ Mohamed Y Mustafa, ${ }^{2}$ Jason Knight, ${ }^{1}$ Muhammad Virk $\&{ }^{2}$ George Haritos \\ ${ }^{1}$ Atmospheric Icing Research Team, Narvik University College, 8505 Narvik Norway \\ ${ }^{2}$ School of Engineering \& Technology, University of Hertfordshire, Hatfield, United Kingdom \\ ${ }^{1}$ Email: Yizhong.Xu@hin.no
}

\begin{abstract}
Numerical simulation has played an important role in systematic study on the performance of porous fences over the last three decades. In this paper, a 3D CFD model to simulate air flow through a porous fence is presented. The simulated velocity data has been tested against experimental data acquired in wind tunnel experiments under the same conditions. Good agreement has been observed between the numerical and the experimental results within the fence effective zone, demonstrating that the 3D CFD model presented in the paper is generally sound and can be used to systematically assess the performance of porous fences. It is the first step to create a CogInfocom channel through which a numerical system (CFD) has communicated with a natural cognitive system (prototype and physical experimental system), in a way that improves human cognitive abilities to comprehend air flow through porous fences.
\end{abstract}

Keywords_porous fence; $3 D$ CFD simulation; wind tunnel experiment; CogInfoCom

\section{INTRODUCTION}

Porous fence is an artificial device, which has been widely in application to improve windy and snowy climatic conditions for human needs and animal welfare. In cold regions like Norway, especially with its ever expanding petroleum industry, there is a strong need to design an optimum porous fence system to protect oil workers and machinery from harsh climatic conditions [1].

A porous fence is usually constructed to have optical porosities greater than zero, so that it produces artificial windbreaks and blocks drifting sediments such as snow. Systematic study on porous fence has been carried out since 1940s. The majority of physical experiments to investigate air flow through porous fences have been conducted using wind tunnel facilities [2] [3] [4] [5]. However, physical testing in wind tunnel has many drawbacks such as: demand for elaborate equipment, limited testing space, extensive man power and time, which eventually lead to high cost. Moreover, conventional measurement techniques may interfere with the wind field and therefore produce measurement discrepancies. Hence, it remains difficult to obtain proper comprehension of the wind flow behavior near the fence zone through wind tunnel experiments due to the complexity of turbulence and the lack of a quantitative theory. With the rapid development of computer technology and Computational Fluid Dynamics
(CFD) techniques, CFD simulation, as a flexible, efficient and relatively cheap alternative to physical experiment, has been successfully and increasingly applied in porous fence researches over the last three decades [6] [7] [8].

Considerable CFD simulations in the field are performed by 2D models. However, 2D simulations are unable to perfectly reflect the full structure of air flow in the simulation domain. Besides, they require strict modifications of operating and boundary conditions, and they vary almost in each individual case, which increases uncertainty of numerical results.

CFD techniques, particularly for 3D CFD model simulation, can provide a comprehensive structure of air flow behind a porous fence, which reflects its prototype, if the numerical model is proved to be sound. Furthermore, CFD is convenient for re-defining porous fences, the related domain, operating and boundary conditions, which involves great advantages for evaluating the performance of porous fence (under different conditions) and optimizing the design of porous fences. It is considered as a part of CogInfoCom research activities, since it has created a CogInfoCom channel through which an artificially cognitive system (CFD system) has communicated with a natural cognitive system (prototype and physical experimental system) in a way that improves the cognitive abilities for researchers in the field. [9].

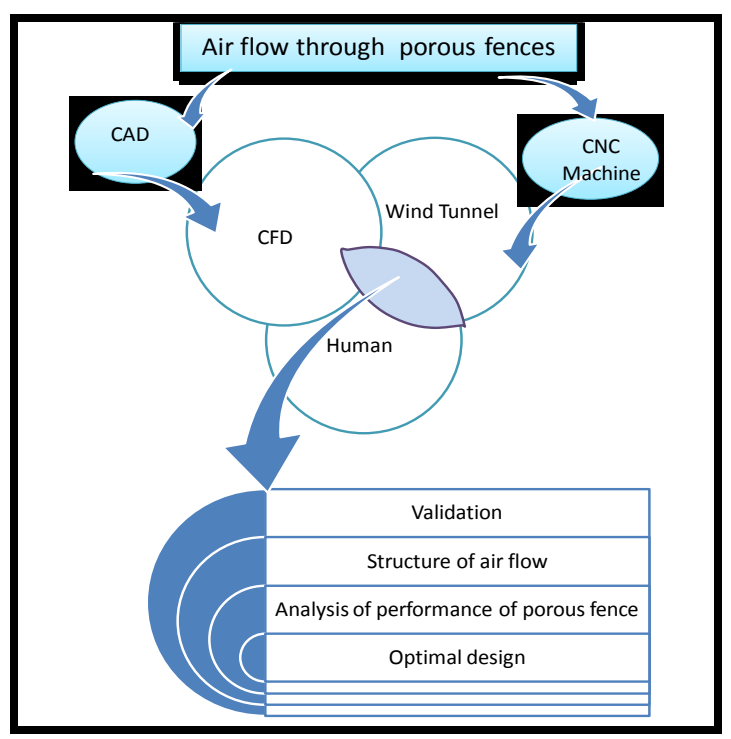

Fig. 1: CogInfoCom process of air flow through a porous fence 
Fig.1 demonstrates a schematic of the CogInfoCom process implemented in this research. For the CogInfoCom process to move correctly in the forward direction, the numerical model has to be proved sound in the first place. This paper presents a 3D CFD simulation model to study air flow through a porous fence. To validate the numerical model, the simulated velocity data are tested against experimental data obtained through wind tunnel experiments under the same conditions.

\section{GEOMETRIC 3D CFD MODEL ESTABLISHMENT}

The 3D CFD simulation has been performed using ANSYS14.0 Fluent workbench package. In order to validate simulation results against experimental results, the configurations of the CFD domain and fence model are the same as those of the wind tunnel and the fence test sample. Fig. 2 shows the 3D domain with height*length = $655^{*} 4000 \mathrm{~mm}^{2}$, and its maximum width is $1160 \mathrm{~mm}$. The fence, which is at a distance of $1000 \mathrm{~mm}$ from the leading edge of the domain (upstream), is parallel to and centered at the cross section of the domain. The volumetric configuration of the fence is width*height $*$ thickness $=650 * 200 * 3 \mathrm{~mm}^{3}$. It is an oval holed fence with the porosity of 0.23 . Blockage ratio of the wind tunnel therefore is $9.8 \%$.

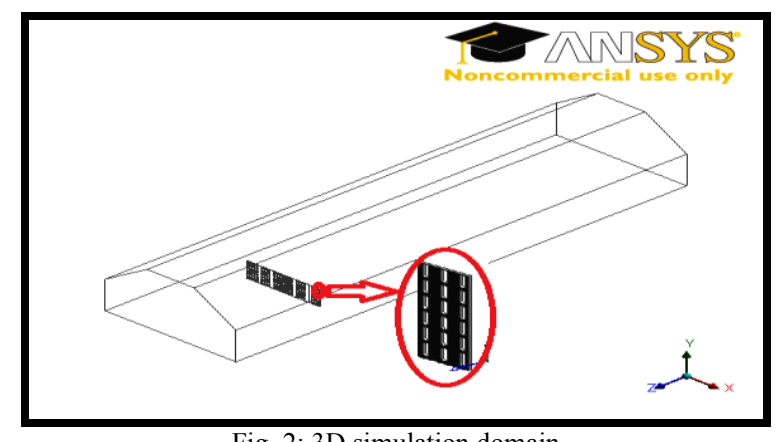

Fig. 2: 3D simulation domain

\section{RESULTS AND DISCUSSIONS}

\section{A. Mesh and mesh sensitivity analysis}

To save simulation time, and due to the fact that the meshed domain is symmetrical in the YZ plane, only half of the domain is considered in the simulation. Furthermore, to reduce the quantity of meshed elements, an air box $\left(\right.$ Length $*$ width $*$ height $=3000 * 400 * 300 \mathrm{~mm}^{3}$ with upstream length of $500 \mathrm{~mm}$ ) has been created in the most influential zone of the fence. Using the 'Body of Influence' function in order to re-define the element size within the air box, and using 'edge sizing' to refine the elements around the oval holes.

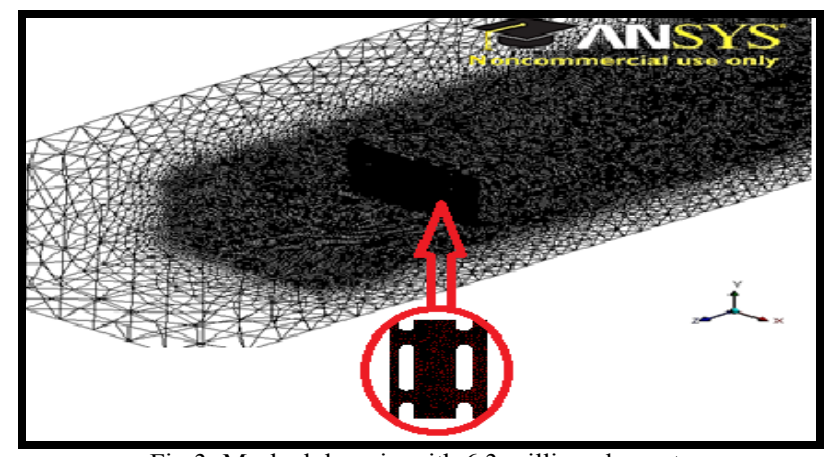

Fig.3: Meshed domain with 6.3 million elements
Fig. 3 demonstrates the meshed domain with 6.3million elements. Under the same simulation conditions, the mesh sensitivity has been investigated through 6 different meshes (see Table I). By analyzing the outputs of velocity magnitude and turbulent kinetic energy (TKE), the mesh with 6.3 million elements has reached grid independence, and therefore it is considered to be successful.

Table I: List of different meshing methods

\begin{tabular}{|c|c|c|c|}
\hline \multicolumn{2}{|c|}{ Meshing methods } \\
\hline $\begin{array}{c}\text { Mesh } \\
\text { No. }\end{array}$ & Element & Ele. Type & Method \\
\hline No.1 & 622,963 & Tetrahedral & Default mesh \\
\hline No.2 & $1,192,181$ & Tetrahedral & Default mesh with relevance at 50 \\
\hline No.1 & $4,073,281$ & Tetrahedral & Body \&edge sizing with face mapping \\
\hline No.2 & $5,322,127$ & Tetrahedral & Body \&edge sizing with face mapping \\
\hline No.3 & $6,280,837$ & Tetrahedral & Body \&edge sizing with face mapping \\
\hline No.4 & $7,209,309$ & Tetrahedral & Body \&edge sizing with face mapping \\
\hline
\end{tabular}

\section{B. Numerical simulation}

In the wind shielding industry, k-Epsilon and k-Omega are the two most popular turbulence models in numerical simulations [8] [10] [6] [11] [12]. The k-Epsilon model solves kinetic energy and turbulent dissipation, while the k-Omega model solves kinetic energy and turbulent frequency. Both of the two turbulence models have been successfully employed in CFD simulations. It is generally recognized that the standard kEpsilon model over-predicts the production of turbulent kinetic energy in the flow with bluff bodies and separated flow, while Re-Normalization Group (RNG) and realizable kEpsilon models are in remarkable agreement with the testing results. In particular, realizable k-Epsilon model works even better [13].

The CFD simulations in this paper use the realizable k-Epsilon turbulence model with Non-Equilibrium Wall Functions for near-wall treatment. The solution method is the pressurevelocity coupling the SIMPLE (Semi-Implicit Method for Pressure-Linked Equation) scheme. The SIMPLE algorithm is adequate as it has been extensively used for atmospheric flows, which is compatible with the environment of offshore platforms [11] [13] since the targeted application of the tested fence is on offshore oil and gas facilities.

The CFD simulation is implemented to investigate the flow behavior behind the fence under free stream velocities of $15 \mathrm{~m} / \mathrm{s}$ and $20 \mathrm{~m} / \mathrm{s}$ respectively. Turbulent intensity ratio and viscosity ratio at the velocity inlet are set to $1 \%$ and $10 \%$ respectively. Gauge pressure at the pressure outlet is set to 0 Pascal with backflow turbulent intensity ratio and viscosity ratio as $5 \%$ and $10 \%$ respectively. All of the rest boundary conditions are treated as no-slip stationary walls with $\mathrm{Om}$ roughness height and 0.5 roughness constant. The monitors 
use scaled residuals with convergence criteria below $1 * 10^{-4}$. To avoid numerical error, the Second Order Upwind method has been performed in each case.

\section{Validation against wind tunnel tests}

\section{1) Wind tunnel experiment}

The wind tunnel experiment in this paper is designed to investigate velocity reductions behind the fence under free upstream velocities of $15 \mathrm{~m} / \mathrm{s}$ and $20 \mathrm{~m} / \mathrm{s}$ respectively. In the experiment, a Pitot static tube is placed at the entrance of the test section to monitor the upstream velocity, and a traverse attached with a Hot-Wire- Anemometer (HWA) is placed at a longitudinal distance (from the fence) of $925 \mathrm{~mm}$ downstream. Data are taken by moving the traverse at steps of 0.2 inch in the vertical direction. To improve the accuracy of the results, 50 readings have been taken for each step, and then their mathematical average was taken. Hence, the obtained data are the mean velocities in the longitudinal direction (Z-Axis) along the vertical direction (Y-Axis).

Before the fence was put in position, the velocity profiles at the fence position have been measured and Reynolds numbers and boundary thicknesses have been checked. The results reveal that the boundary layer in the experiment is compatible to the Atmospheric boundary layer and the velocity profiles appear to be algorithmic. Also, velocity profiles at the measurement point have also been checked by a Hot-WireAnemometer (HWA) for future reference.

\section{2) Validating CFD results against experimental results} Validating CFD results against experimental results is strongly required as the numerical simulation is based on some assumptions, since numerical approximations, parameterization schemes (e.g. turbulence models, discretization techniques) and the choice of boundary conditions can introduce errors in simulated data sets.

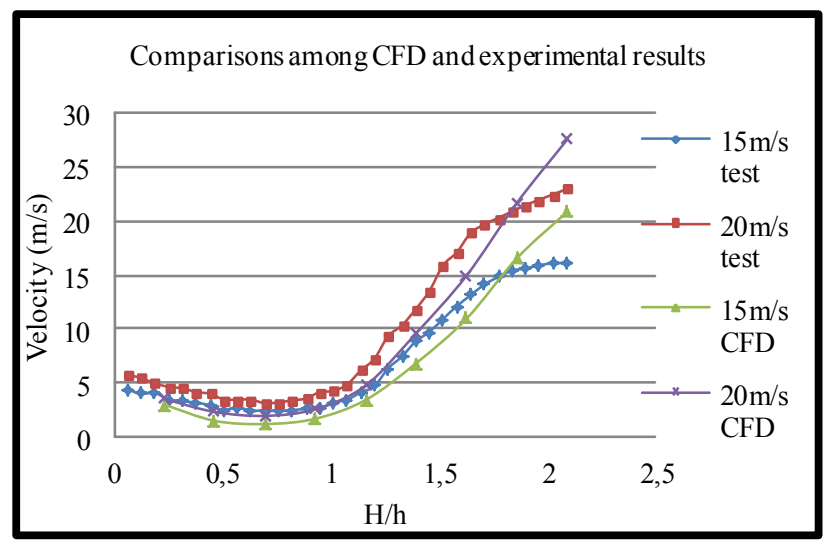

Fig.4: Comparisons among CFD and experimental results

Experimentation in the wind tunnel has revealed that the boundary layer is compatible to Atmospheric Boundary Layer. As such, the no slip stationary wall is an acceptable wall treatment in the simulations. The numerical domain and fence model are identical to the physical ones. Overall, the numerical model and boundary condition treatments are representative of the wind tunnel experiment and the simulation results therefore can be comparable to the experimental ones.

The comparing numerical data set is taken from the line at points $(0,-0.1,-0.925 \mathrm{~mm})$ and $(0,0.32,-0.925 \mathrm{~mm})$, and the plotted velocities are the Z-Axial velocities. By adjusting its coordinates, it is the exact position of the experimental measurement position. Fig. 4 shows the comparisons between the numerical simulation and the wind tunnel testing results, where $H / h$ is the ratio of the measuring height to the fence height.

\section{Discussions}

The following conclusions can be drawn from this work:

1) The 3D CFD results are in good agreement with the experimental results especially within the range of $\mathrm{H} / \mathrm{h}=$ 1.2. In the range between $H / h=1.2$ and $H / h=1.8$, the discrepancy between CFD and experimental results tends to increase. However, the inclinations remain similar. Beyond the range of $\mathrm{H} / \mathrm{h}=1.8$, the velocities in CFD grow faster than those in the experiment. The reason can be attributed to the fact that wind tunnel blockage ratio in the current setting is slightly high, which increases the effects of the top wall boundary layer on the regional velocities in the wind tunnel test, while in the case of CFD, the roughness height of the top wall is set to 0 ;

2) The CFD simulation over-predicted the reduction of velocity when compared to wind tunnel results. However, the CFD results are still promising. The discrepancy between CFD and experiment is around 20\% in general, which is at the boundaries of acceptable limits.

3) Fig. 5 and Fig.6 are the structures of velocity magnitude and the kinetic energy on 3 planes obtained from the numerical simulation, where the free stream velocity is at $20 \mathrm{~m} / \mathrm{s}$; Plane-1 is parallel to the symmetry wall with $\mathrm{X}=0.011 \mathrm{~m}$; Plane-2 is parallel to the floor with $\mathrm{Y}=0 \mathrm{~m}$; and Plane-3 is parallel to the velocity inlet with $Z=-1.5 \mathrm{~m}$. The $3 \mathrm{D}$ numerical simulation can provide comprehensive information to scrutinize the characteristics of air flow within the simulation domain, some of which may be difficult to obtain under the current experimental set-up, such as the full structure of turbulence, shear distribution in the porous fence zone, etc. Moreover, it is easy to manipulate the domain structure in the 3D model (i.e. domain, fence, porosity, shape of pore, etc.), in order to exercise different turbulence models, and to switch to multi-phase flow.

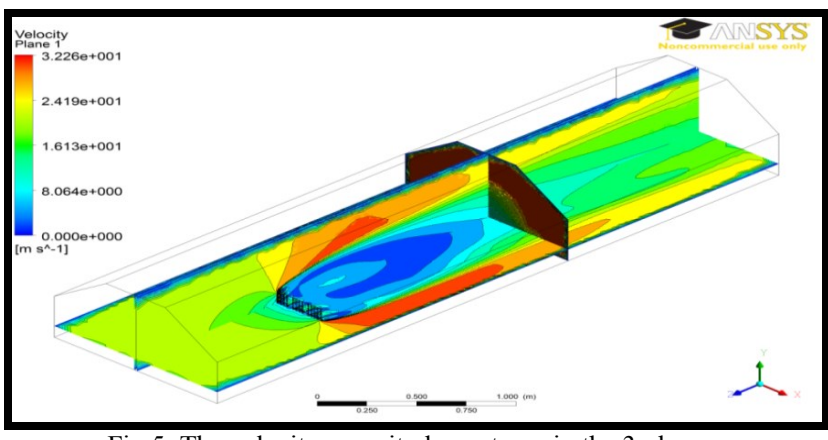

Fig.5: The velocity magnitude contours in the 3 planes 


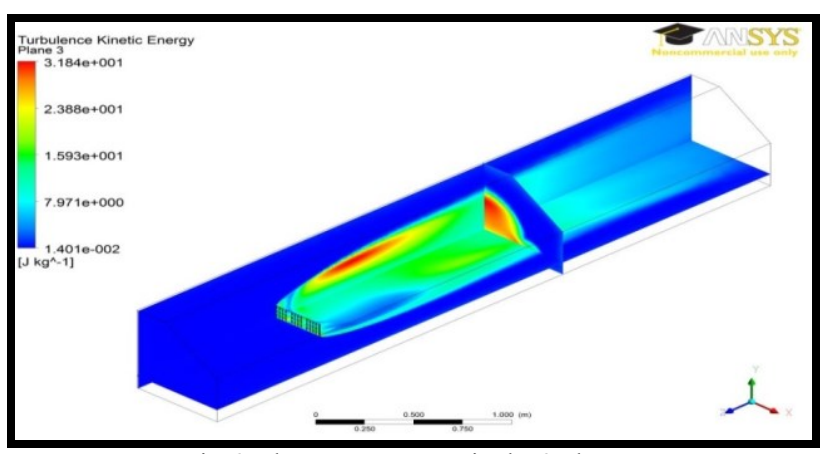

Fig.6: The TKE contours in the 3 planes

\section{CONCLUSIONS}

The following conclusions can be drawn from this study:

- Detailed set-up of the CFD model to investigate the wind flow behind a porous wind fence has been presented in the paper.

- CFD simulation results on velocity magnitudes have been obtained. A good agreement has been found between the simulation and the experimental results. The CFD model has been proved to be sound and is valid.

- It is an increasingly adapted research approach to study air flow behind porous fence by implementing CFD techniques together with wind tunnel techniques. CFD can overcome the limitations and weaknesses of wind tunnel experiments with flexibility, efficiency and low cost, and can help to improve human cognition of the problems. The CFD simulation is a part of the CogInfoCom research activities in the field.

\section{ACKNOWLEDGMENT}

This work was financed by Norwegian Research Council under project number 195153 (ColdTech). The authors would also like to acknowledge the contribution of the industrial partner in this research task: IKM dsc AS, Norway.

\section{REFERENCES}

[1] X. Yizhong, V. Muhammad S., K. Jason, M. Mohamad Y. and H. George, "Factors influencing the performance of porous wind shields," Applied Mechanics and Materials, Vols. 321-324, pp. 799-803, 2013.

[2] N. Tani, "On the wind tunnel test of the model shelter hedge," in Bulletin of the Natinal Institute for Agricultural Sciences, 1958.

[3] R. Bagnold, The physics of blown sand and desert dunes, London, 1941.

[4] J. Raine and D. Stevenson, "Wind protection by model fences in simulated atmospheric boundary layer," Industrial Aerodynamics, vol. 2, pp. 159-180, 1977.

[5] W. Cornelis and D. Gabriels, "Optimal windbreak design for wind-erosion control," Arid Enviroment, vol. 61, pp. 315-332, 2005.

[6] J. Wilson, "Numerical studies of flow through a windbreak," Wind Eng. Ind. Aerodyn, vol. 21, pp. 119154,1985

[7] A. S., "Comutational fluid dynamic modelling of drifting particles at porous fence," Environmental Modelling and Software, vol. 19, pp. 163-170, 2004.

[8] P. A., "Flow through porous fences in thick boundary layers: comparisons between laboratory and numerical experiments," Wind Eng. Ind. Aerodyn., vol. 88, pp. 7590,2000 .

[9] B. Peter and C. Adam, "Definition and synergies of cognitive infocommunications," Acta Polytechnica Hungarica, vol. 9, no. 1, pp. 67-83, 2012.

[10] P. Richards and R. Hoxey, "Appropriate boundary conditions for computational wind engineering models using the k-e turbulence model," Wind Eng. Ind. Aerodyn., vol. 46 (47), pp. 145-153, 1993.

[11] J. Wilson and C. Mooney, "A numerical simulation of boundary-layer flows near shelterbelts comments," Boundary-Layer Meteorology, vol. 85, pp. 137-149, 1997.

[12] G. C., W. W., C. S. and J. L., "3D numerical simulation of wind blow behind a new porous fence," Powder Technology, pp. PTEC-09046, 2012.

[13] S. J.L., M. F., B. N. and S.-A. A., "Experimental and numerical study of wind flow behind windbreaks," Atmospheric Environ., vol. 41, pp. 6406-6420, 2007. 